\title{
Anti-periodic solutions for neutral type FCNNs with time-varying delays and D operator on time scales
}

\author{
Bing $\mathrm{Li}^{\mathrm{a}}$, Yongkun Lij,* \\ a School of Mathematics and Computer Science, Yunnan Nationalities University, Kunming, Yunnan 650500, People's Republic of China. \\ ${ }^{b}$ Department of Mathematics, Yunnan University, Kunming, Yunnan 650091, People's Republic of China.
}

Communicated by A. Atangana

\begin{abstract}
In this paper, we consider a class of neutral type fuzzy cellular neural networks with time-varying delays and D operator on time scales. Based on inequality analysis techniques on time scales and a fixed point theorem and the theory of calculus on time scales, we obtain the existence and global exponential stability of anti-periodic solutions for this class of the networks. Finally, a numerical example is given to illustrate the feasibility of our results. (C)2017 All rights reserved.
\end{abstract}

Keywords: Fuzzy cellular neural networks, anti-periodic solution, D operator, global exponential stability, time scales. 2010 MSC: 34K13, 34K36, 92B20, 34N05.

\section{Introduction}

So far, there are two basic cellular neural networks (CNNs) structures being proposed. The first one is traditional CNNs ([4,5]). The second one is fuzzy cellular neural networks (FCNNs) ([17, 18]). Unlike the first structure, FCNNs has fuzzy logic between its template and input and/or output besides the sum of product operations, which allows us to combine the low of fuzzy systems. FCNNs is very useful paradigm for image processing problems, which is a cornerstone in image processing and pattern recognition. In recent years, various interesting results on the dynamical behaviors of FCNNs with delays have been reported $[1,2,6,7,10-16,19,20,22,23]$.

On the other hand, according to the theory of functional differential equations, neutral type CNNs with D operator may have more realistic significance than non-operator-based ones in many practical applications of neural networks' dynamics. However, among the existing results about the dynamics of FCNNs, most of them did not consider the effect of neutral type delays. So, the dynamics of neutral type FCNNs with D operator should be further studied. Moreover, although the signal transmission process of neural networks can often be regarded as an anti-periodic process, in particular, voltage transmission process of CNNs have an anti-periodic process, the periodic dynamics of neural networks models with

\footnotetext{
*Corresponding author

Email addresses: bli123@126.com (Bing Li), yklie@ynu.edu.cn (Yongkun Li)
} 
D operator has been extensively investigated (see $[2,15,19]$ and the references cited therein), the antiperiodic one remains less touched.

In addition, it is well-known that both continuous time and discrete time neural networks are very important in implementation and applications. But, few results are available for discrete time FCNNs which are more convenient for numerical simulations than the continuous ones. Besides, in fact, studying neural networks can unify the continuous time case and the discrete time case $([3,8])$. However, to the best of our knowledge, there is no published paper considering the anti-periodic solutions for FCNNs with time-varying delays and D operator on time scales. Therefore, it is very important in theories and applications and also is a very challenging problem.

Motivated by the above statement, in this paper, we are concerned with the following neutral type FCNNs with time-varying delays and D operator on time scales

$$
\begin{aligned}
{\left[x_{i}(t)-c_{i}(t) x_{i}\left(t-\sigma_{i}(t)\right)\right]^{\Delta}=} & -a_{i}(t) x_{i}(t)+\sum_{j=1}^{n} b_{i j}(t) f_{j}\left(x_{j}\left(t-\tau_{i j}(t)\right)\right)+\sum_{j=1}^{n} d_{i j}(t) \mu_{j}(t) \\
& +\bigwedge_{j=1}^{n} \alpha_{i j}(t) \int_{t-\eta_{i j}(t)}^{t} g_{j}\left(x_{j}(s)\right) \Delta s+\bigvee_{j=1}^{n} \beta_{i j}(t) \int_{t-\xi_{i j}(t)}^{t} g_{j}\left(x_{j}(s)\right) \Delta s \\
& +\bigwedge_{j=1}^{n} T_{i j}(t) \mu_{j}(t)+\bigvee_{j=1}^{n} S_{i j}(t) \mu_{j}(t)+I_{i}(t), \quad t \in \mathbb{T}, \quad i=1,2, \cdots, n,
\end{aligned}
$$

where $\mathbb{T}$ is a translation invariant time scale, $n$ is the number of neurons in layers, $x_{i}(t)$ denotes the activations of the $i$-th neuron at time $t, a_{i}$ represents the rate with which the $i$-th neuron will reset its potential to the resting state in isolation when they are disconnected from the network and the external inputs at time $t, \alpha_{i j}, \beta_{i j}, T_{i j}$ and $S_{i j}$ are the elements of fuzzy feedback MIN template, fuzzy feedback MAX template, fuzzy feed forward MIN template and fuzzy feed forward MAX template, respectively, $b_{i j}$ and $d_{i j}$ are the elements of feedback template and feed forward template, $\Lambda, \vee$ denote the fuzzy AND and fuzzy OR operations, respectively, $f_{j}$ and $g_{j}$ are the activation functions, $\sigma_{i}(t), \tau_{i j}(t), \eta_{i j}(t)$ and $\xi_{i j}(t)$ are transmission delays at time $t$ and satisfy $t-\sigma_{i}(t) \in \mathbb{T}, t-\tau_{i j}(t) \in \mathbb{T}, t-\eta_{i j}(t) \in \mathbb{T}$ and $t-\xi_{i j}(t) \in \mathbb{T}$ for $t \in \mathbb{T}, I_{i}$ denotes the input of the $i$-th neuron at time $t, i, j=1,2, \cdots, n$.

The initial conditions associated with system (1.1) are of the form

$$
x_{i}(s)=\varphi_{i}(s), \quad s \in[-\theta, 0]_{\mathbb{T}},
$$

where $\theta=\sup _{t \in \mathbb{T}}\left\{\max _{1 \leqslant i \leqslant n}\left\{\sigma_{i}(t)\right\}, \max _{1 \leqslant i, j \leqslant n}\left\{\tau_{i j}(t), \eta_{i j}(t), \xi_{i j}(t)\right\}\right\}, \varphi_{i} \in C\left([-\theta, 0]_{\mathbb{T}}, \mathbb{R}^{+}\right), i=1,2, \cdots, n$.

Throughout this paper, we denote $[a, b]_{\mathbb{T}}=[a, b] \cap \mathbb{T}$. For convenience, for an anti-periodic function $f: \mathbb{T} \rightarrow \mathbb{R}$, we denote $f^{-}=\inf _{t \in \mathbb{T}}|f(t)|$ and $f^{+}=\sup _{t \in \mathbb{T}}|f(t)|$.

This paper is organized as follows. In Section 2, we introduce some definitions and preliminary lemmas. In Section 3, we derive some sufficient conditions for the existence and global exponential stability of anti-periodic solutions of (1.1). In Section 4, we give an example to demonstrate the feasibility of our results. This paper ends with a brief conclusion in Section 5.

\section{Preliminaries}

In this section, we shall recall some basic definitions, lemmas which are used in what follows.

A time scale $\mathbb{T}$ is an arbitrary nonempty closed subset of the real numbers, the forward and backward jump operators $\sigma, \rho: \mathbb{T} \rightarrow \mathbb{T}$ and the forward graininess $\mu: \mathbb{T} \rightarrow \mathbb{R}^{+}$are defined, respectively, by $\sigma(t):=\inf \{s \in \mathbb{T}: s>t\}, \rho(t):=\sup \{s \in \mathbb{T}: s<t\}$ and $\mu(t)=\sigma(t)-t$. A point $t$ is said to be left-dense if $t>\inf \mathbb{T}$ and $\rho(t)=t$, right-dense if $t<\sup \mathbb{T}$ and $\sigma(t)=t$, left-scattered if $\rho(t)<t$ and right-scattered if $\sigma(t)>t$. 
A function $f: \mathbb{T} \rightarrow \mathbb{R}$ is right-dense continuous or rd-continuous provided it is continuous at rightdense points in $\mathbb{T}$ and its left-sided limits exist (finite) at left-dense points in $\mathbb{T}$. If $f$ is continuous at each right-dense point and each left-dense point, then $f$ is said to be a continuous function on $\mathbb{T}$.

A function $p: \mathbb{T} \rightarrow \mathbb{R}$ is called regressive provided $1+\mu(t) p(t) \neq 0$ for all $t \in \mathbb{T}^{k}$. The set of all regressive and rd-continuous functions $p: \mathbb{T} \rightarrow \mathbb{R}$ will be denoted by $\mathcal{R}=\mathcal{R}(\mathbb{T})=\mathcal{R}(\mathbb{T}, \mathbb{R})$. We define the set $\mathcal{R}^{+}=\mathcal{R}^{+}(\mathbb{T}, \mathbb{R})=\{\mathrm{p} \in \mathcal{R}: 1+\mu(\mathrm{t}) \mathrm{p}(\mathrm{t})>0, \forall \mathrm{t} \in \mathbb{T}\}$.

Lemma 2.1 ([16]). Suppose $x$ and $y$ are two states of system (1.1). Then we have

$$
\begin{aligned}
& \left|\bigwedge_{j=1}^{n} \alpha_{i j}(t) f_{j}(x)-\bigwedge_{j=1}^{n} \alpha_{i j}(t) f_{j}(y)\right| \leqslant \sum_{j=1}^{n}\left|\alpha_{i j}(t)\right|\left|f_{j}(x)-f_{j}(y)\right|, \\
& \left|\bigvee_{j=1}^{n} \beta_{i j}(t) f_{j}(x)-\bigvee_{j=1}^{n} \beta_{i j}(t) f_{j}(y)\right| \leqslant \sum_{j=1}^{n}\left|\beta_{i j}(t)\right|\left|f_{j}(x)-f_{j}(y)\right|,
\end{aligned}
$$

where $i=1,2, \cdots, n$.

Definition 2.2 ([9]). A time scale $\mathbb{T}$ is called a translation invariant time scale if

$$
\Pi:=\{\tau \in \mathbb{R}: t \pm \tau \in \mathbb{T}, \forall \mathrm{t} \in \mathbb{T}\} \neq\{0\} .
$$

Definition 2.3. Let $\mathbb{T} \neq \mathbb{R}$ be a translation invariant time scale with $p=\inf \{\tau \in \Pi\}>0$. We say that the function $f: \mathbb{T} \rightarrow \mathbb{R}$ is periodic with period $\omega$, if there exists a natural number $n$ such that $\omega=n p, f(t+\omega)=f(t)$ for all $t \in \mathbb{T}$ and $\omega$ is the smallest number such that $f(t+\omega)=f(t)$ and we say that the function $f: \mathbb{T} \rightarrow \mathbb{R}$ is anti-periodic with period $\omega$, if there exists a natural number $n$ such that $\omega=n p, f(t+\omega)=-f(t)$ for all $t \in \mathbb{T}$ and $\omega$ is the smallest number such that $f(t+\omega)=-f(t)$.

If $\mathbb{T}=\mathbb{R}$, we say that $f$ is periodic with period $\omega>0$ if $\omega$ is the smallest positive number such that $f(t+\omega)=f(t)$ for all $t \in \mathbb{T}$ and we say that $f$ is periodic with period $\omega>0$ if $\omega$ is the smallest positive number such that $f(t+\omega)=-f(t)$ for all $t \in \mathbb{T}$.

Lemma 2.4 ([21]). Let $\mathbb{T}$ be a translation invariant time scale and $\mathrm{h} \in \Pi$. Then

(i) $\sigma(t+h)=\sigma(t)+h$ and $\sigma(t-h)=\sigma(t)-h$, for every $t \in \mathbb{T}$;

(ii) $\mu(t+h)=\mu(t)=\sigma(t-h)$, for every $t \in \mathbb{T}$.

\section{Main results}

In this section, we will state and prove the sufficient conditions for the existence and global exponential stability of anti-periodic solutions of (1.1).

Set $\mathbb{X}=\left\{\varphi \in \mathrm{C}\left(\mathbb{T}, \mathbb{R}^{\mathfrak{n}}\right): \varphi(\mathrm{t}+\omega)=-\varphi(\mathrm{t})\right\}$ with the norm $\|\varphi\|_{\mathbb{X}}=\sup _{\mathrm{t} \in \mathbb{T}}|\varphi(\mathrm{t})|$, then $\mathbb{X}$ is a real Banach space. Let $\varphi^{0}=\left(\varphi_{1}^{0}, \varphi_{2}^{0}, \cdots, \varphi_{\mathrm{n}}^{0}\right)^{\mathrm{T}}$, where

$$
\varphi_{i}^{0}(t)=\int_{-\infty}^{t} e_{-a_{i}}(t, \sigma(s))\left(\sum_{j=1}^{n} d_{i j}(s) \mu_{j}(s)+\bigwedge_{j=1}^{n} T_{i j}(s) \mu_{j}(s)+\bigvee_{j=1}^{n} s_{i j}(s) \mu_{j}(s)+I_{i}(s)\right) \Delta s, i=1,2, \cdots, n,
$$

and $L$ be a constant satisfying $L \geqslant\left\|\varphi^{0}\right\|_{\mathbb{X}}$.

Throughout this paper, we assume that the following conditions hold:

$\left(\mathrm{H}_{1}\right)-\mathrm{a}_{i} \in \mathcal{R}, \mathrm{a}_{i}, \sigma_{i}, \tau_{i j}, \eta_{i j}, \xi_{i j} \in \mathrm{C}\left(\mathbb{T}, \mathbb{R}^{+}\right), c_{i}, b_{i j}, \mathrm{~d}_{i j}, \alpha_{i j}, \beta_{i j}, \mathrm{~T}_{i j}, \mathrm{~S}_{i j} \in \mathrm{C}(\mathbb{T}, \mathbb{R})$ are $\omega$-periodic, and $\mu_{j}, I_{i} \in C(\mathbb{T}, \mathbb{R})$ are $\omega$-anti-periodic, where $i, j=1,2, \cdots, n$; 
$\left(H_{2}\right) f_{j}, g_{j} \in C(\mathbb{R}, \mathbb{R}), f_{j}(u)=-f_{j}(-u), g_{j}(u)=-g_{j}(-u)$ for all $u \in \mathbb{R}$ and there exist positive constants $L_{j}^{f}$ and $L_{j}^{g}$ such that $\left|f_{j}(x)-f_{j}(y)\right| \leqslant L_{j}^{f}|x-y|,\left|g_{j}(x)-g_{j}(y)\right| \leqslant L_{j}^{g}|x-y|$ for all $x, y \in \mathbb{R}, j=1,2, \cdots, n$;

$\left(H_{3}\right) \rho=\max _{1 \leqslant i \leqslant n}\left\{c_{i}^{+}+\frac{1}{a_{i}^{-}}\left(a_{i}^{+} c_{i}^{+}+\sum_{j=1}^{n}\left(L_{j}^{f} b_{i j}^{+}+L_{j}^{g} \alpha_{i j}^{+} \eta_{i j}^{+}+L_{j}^{g} \beta_{i j}^{+} \xi_{i j}^{+}\right)\right)\right\}<1, i=1,2, \cdots, n$.

Theorem 3.1. Let $\left(\mathrm{H}_{1}\right)-\left(\mathrm{H}_{3}\right)$ hold. Then, system (1.1) has an anti-periodic solution in

$$
\mathbb{X}^{*}=\left\{\varphi \in \mathbb{X} \mid\left\|\varphi-\varphi^{0}\right\|_{\mathbb{X}} \leqslant \frac{\rho \mathrm{L}}{1-\rho}\right\} .
$$

Proof. Let

$$
Y_{i}(t)=x_{i}(t)-c_{i}(t) x_{i}\left(t-\sigma_{i}(t)\right)
$$

Then

$$
\begin{aligned}
Y_{i}^{\Delta}(t)= & {\left[x_{i}(t)-c_{i}(t) x_{i}\left(t-\sigma_{i}(t)\right)\right]^{\Delta} } \\
= & -a_{i}(t) Y(t)-a_{i}(t) c_{i}(t) x_{i}\left(t-\sigma_{i}(t)\right) \\
& +\sum_{j=1}^{n} b_{i j}(t) f_{j}\left(x_{j}\left(t-\tau_{i j}(t)\right)\right)+\sum_{j=1}^{n} d_{i j}(t) \mu_{j}(t) \\
& +\bigwedge_{j=1}^{n} \alpha_{i j}(t) \int_{t-\eta_{i j}(t)}^{t} g_{j}\left(x_{j}(s)\right) \Delta s+\bigvee_{j=1}^{n} \beta_{i j}(t) \int_{t-\xi_{i j}(t)}^{t} g_{j}\left(x_{j}(s)\right) \Delta s \\
& +\bigwedge_{j=1}^{n} T_{i j}(t) \mu_{j}(t)+\bigvee_{j=1}^{n} s_{i j}(t) \mu_{j}(t)+I_{i}(t), \quad i=1,2, \cdots, n .
\end{aligned}
$$

For any $\varphi \in \mathbb{X}^{*}$, we consider the following anti-periodic system:

$$
Y_{i}^{\Delta}(t)=-a_{i}(t) Y_{i}(t)+F_{i}(t, \varphi)+I_{i}(t),
$$

where

$$
\begin{aligned}
F_{i}(t, \varphi)= & -a_{i}(t) c_{i}(t) \varphi_{i}\left(t-\sigma_{i}(t)\right)+\sum_{j=1}^{n} b_{i j}(t) f_{j}\left(\varphi_{j}\left(t-\tau_{i j}(t)\right)\right)+\sum_{j=1}^{n} d_{i j}(t) \mu_{j}(t) \\
& +\bigwedge_{j=1}^{n} \alpha_{i j}(t) \int_{t-\eta_{i j}(t)}^{t} g_{j}\left(\varphi_{j}(s)\right) \Delta s+\bigvee_{j=1}^{n} \beta_{i j}(t) \int_{t-\xi_{i j}(t)}^{t} g_{j}\left(\varphi_{j}(s)\right) \Delta s \\
& +\bigwedge_{j=1}^{n} T_{i j}(t) \mu_{j}(t)+\bigvee_{j=1}^{n} S_{i j}(t) \mu_{j}(t), \quad i=1,2, \cdots, n .
\end{aligned}
$$

Define

$$
Y^{\varphi}(t)=\left(Y_{1}^{\varphi}(t), Y_{2}^{\varphi}(t), \cdots, Y_{n}^{\varphi}(t)\right)^{\top}
$$

where

$$
Y_{i}^{\varphi}(t)=\int_{-\infty}^{t} e_{-a_{i}}(t, \sigma(s))\left(F_{i}(s, \varphi)+I_{i}(s)\right) \Delta s, \quad i=1,2, \cdots, n .
$$

For $\phi \in \mathbb{X}$, we have

$$
Y_{i}^{\phi}(t+T)=\int_{-\infty}^{t+T} e_{-a_{i}}(t+T, \sigma(s))\left(F_{i}(s, \phi)+I_{i}(s)\right) \Delta s
$$




$$
\begin{aligned}
& =\int_{-\infty}^{t} e_{-a_{i}}(t+T, \sigma(s+T))\left(F_{i}(s+T, \phi)+I_{i}(s+T)\right) \Delta s \\
& =\int_{-\infty}^{t} e_{-a_{i}}(t+T, \sigma(s)+T)\left(F_{i}(s+T, \phi)+I_{i}(s+T)\right) \Delta s \\
& =-\int_{-\infty}^{t} e_{-a_{i}}(t, \sigma(s))\left(F_{i}(s, \phi)+I_{i}(s)\right) \Delta s, \quad i=1,2, \cdots, n,
\end{aligned}
$$

that is, $\mathrm{Y}^{\Phi} \in \mathbb{X}$. For $\varphi \in \mathbb{X}^{*}$, we find $\|\varphi\|_{\mathbb{X}} \leqslant\left\|\varphi-\varphi^{0}\right\|_{\mathbb{X}}+\left\|\varphi^{0}\right\|_{\mathbb{X}} \leqslant \frac{\rho L}{1-\rho}+\mathrm{L}=\frac{\mathrm{L}}{1-\rho}$.

Define an operator as follows:

$$
\Phi: \mathbb{X}^{*} \rightarrow \mathbb{X}^{*}, \quad\left(\phi_{1}, \phi_{2}, \cdots, \phi_{\mathrm{n}}\right)^{\mathrm{T}} \rightarrow\left((\Phi \phi)_{1},(\Phi \phi)_{2}, \cdots,(\Phi \phi)_{\mathrm{n}}\right)^{\top}
$$

where

$$
(\Phi \phi)_{i}(t)=c_{i}(t) \phi_{i}\left(t-\sigma_{i}(t)\right)+Y_{i}^{\phi}(t), \quad \forall \phi \in \mathbb{X}^{*} .
$$

First we show that for any $\varphi \in \mathbb{X}^{*}, \Phi \varphi \in \mathbb{X}^{*}$. From Lemma 2.1 , for $i=1,2, \cdots, n$, we have

$$
\begin{aligned}
& \left|(\Phi \varphi)_{i}(t)-\varphi_{i}^{0}(t)\right|=\mid c_{i}(t) \varphi_{i}\left(t-\sigma_{i}(t)\right)+\int_{-\infty}^{t} e_{-a_{i}}(t, \sigma(s))\left(-a_{i}(s) c_{i}(s) \varphi_{i}\left(s-\sigma_{i}(s)\right)\right. \\
& +\sum_{j=1}^{n} b_{i j}(s) f_{j}\left(\varphi_{j}\left(s-\tau_{i j}(s)\right)\right)+\bigwedge_{j=1}^{n} \alpha_{i j}(s) \int_{s-\eta_{i j}(s)}^{s} g_{j}\left(\varphi_{j}(u)\right) \Delta u \\
& \left.+\bigvee_{j=1}^{n} \beta_{i j}(s) \int_{s-\xi_{i j}(s)}^{s} g_{j}\left(\varphi_{j}(u)\right) \Delta u\right) \Delta s \\
& \leqslant c_{i}^{+}\left|\varphi_{i}\left(t-\sigma_{i}(t)\right)\right|+\int_{-\infty}^{t} e_{-a_{i}}(t, \sigma(s))\left(a_{i}^{+} c_{i}^{+}\left|\varphi\left(s-\sigma_{i}(s)\right)\right|\right. \\
& +\sum_{j=1}^{n} b_{i j}^{+} L_{j}^{f}\left|\varphi_{j}\left(s-\tau_{i j}(s)\right)\right|+\sum_{j=1}^{n} \alpha_{i j}^{+} \eta_{i j}^{+} L_{j}^{g}\left|\varphi_{j}(s)\right| \\
& \left.+\sum_{j=1}^{n} \beta_{i j}^{+} \xi_{i j}^{+} L_{j}^{g}\left|\varphi_{j}(s)\right|\right) \Delta s \\
& \leqslant c_{i}^{+}\|\varphi\|_{\mathbb{X}}+\int_{-\infty}^{t} e_{-a_{i}}(t, \sigma(s)) \Delta s\left(a_{i}^{+} c_{i}^{+}+\sum_{j=1}^{n} b_{i j}^{+} L_{j}^{f}+\sum_{j=1}^{n} \alpha_{i j}^{+} \eta_{i j}^{+} L_{j}^{g}\right. \\
& \left.+\sum_{j=1}^{n} \beta_{i j}^{+} \xi_{i j}^{+} L_{j}^{g}\right)\|\varphi\|_{\mathbb{X}} \\
& \leqslant c_{i}^{+}\|\varphi\|_{\mathbb{X}}+\frac{1}{a_{i}^{-}}\left(a_{i}^{+} c_{i}^{+}+\sum_{j=1}^{n}\left(L_{j}^{f} b_{i j}^{+}+L_{j}^{g} \alpha_{i j}^{+} \eta_{i j}^{+}+L_{j}^{g} \beta_{i j}^{+} \xi_{i j}^{+}\right)\right)\|\varphi\|_{\mathbb{X}} \\
& \leqslant\left(c_{i}^{+}+\frac{1}{a_{i}^{-}}\left(a_{i}^{+} c_{i}^{+}+\sum_{j=1}^{n}\left(L_{j}^{f} b_{i j}^{+}+L_{j}^{g} \alpha_{i j}^{+} \eta_{i j}^{+}+L_{j}^{g} \beta_{i j}^{+} \xi_{i j}^{+}\right)\right)\right) \frac{L}{1-\rho} \\
& <\frac{\rho \mathrm{L}}{1-\rho}
\end{aligned}
$$

which implies that $\Phi \varphi \in \mathbb{X}^{*}$. Next, we show that $\Phi: \mathbb{X}^{*} \rightarrow \mathbb{X}^{*}$ is a contraction operator. In fact, for any $\varphi=\left(\varphi_{1}, \varphi_{2}, \cdots, \varphi_{n}\right)^{\top}, \psi=\left(\psi_{1}, \psi_{2}, \cdots, \psi_{n}\right)^{\top} \in \mathbb{X}^{*}$, we can get

$$
\left|(\Phi \varphi)_{i}(t)-(\Phi \psi)_{i}(t)\right|=\mid c_{i}(t)\left(\varphi_{i}\left(t-\sigma_{i}(t)\right)-\psi_{i}\left(t-\sigma_{i}(t)\right)\right)+\int_{-\infty}^{t} e_{-a_{i}}(t, \sigma(s))
$$




$$
\begin{aligned}
\times & {\left[-a_{i}(s) c_{i}(s)\left(\varphi_{i}\left(s-\sigma_{i}(s)\right)-\psi_{i}\left(s-\sigma_{i}(s)\right)\right)\right.} \\
+ & \sum_{j=1}^{n} b_{i j}(s)\left(f_{j}\left(\varphi_{j}\left(s-\tau_{i j}(s)\right)\right)-f_{j}\left(\psi_{j}\left(s-\tau_{i j}(s)\right)\right)\right) \\
+ & \bigwedge_{j=1}^{n} \alpha_{i j}(s) \int_{s-\eta_{i j}(s)}^{s}\left(g_{j}\left(\varphi_{j}(u)\right)-g_{j}\left(\psi_{j}(u)\right)\right) \Delta u \\
+ & \left.\bigvee_{j=1}^{n} \beta_{i j}(s) \int_{s-\xi_{i j}(s)}^{s}\left(g_{j}\left(\varphi_{j}(u)\right)-g_{j}\left(\psi_{j}(u)\right)\right) \Delta u\right] \Delta s \mid \\
\leqslant & c_{i}^{+}\left|\varphi_{i}\left(t-\sigma_{i}(t)\right)-\psi_{i}\left(t-\sigma_{i}(t)\right)\right|+\int_{-\infty}^{t} e_{-a_{i}}(t, \sigma(s)) \\
& \times\left[a_{i}^{+} c_{i}^{+}\left|\varphi_{i}\left(s-\sigma_{i}(s)\right)-\psi_{i}\left(s-\sigma_{i}(s)\right)\right|\right. \\
& +\sum_{j=1}^{n} b_{i j}^{+} L_{j}^{f}\left|\varphi_{j}\left(s-\tau_{i j}(s)\right)-\psi_{j}\left(s-\tau_{i j}(s)\right)\right| \\
& \left.+\sum_{j=1}^{n} \alpha_{i j}^{+} \eta_{i j}^{+} L_{j}^{g}\left|\varphi_{j}(s)-\psi_{j}(s)\right|+\sum_{j=1}^{n} \beta_{i j}^{+} \xi_{i j}^{+} L_{j}^{g}\left|\varphi_{j}(s)-\psi_{j}(s)\right|\right] \Delta s \\
\leqslant & \left(c_{i}^{+}+\frac{1}{a_{i}^{-}}\left(a_{i}^{+} c_{i}^{+}+\sum_{j=1}^{n}\left(L_{j}^{f} b_{i j}^{+}+L_{j}^{g} \alpha_{i j}^{+} \eta_{i j}^{+}+L_{j}^{g} \beta_{i j}^{+} \xi_{i j}^{+}\right)\right)\right)\|\varphi-\psi\| \|_{X} \\
< & \rho\|\varphi-\psi\| \|_{X} .
\end{aligned}
$$

Because $\rho<1$, so $\Phi$ is a contraction mapping. By the Banach's fixed point theorem, it follows that $\Phi$ has a fixed point $x^{*}=\left(x_{1}^{*}, x_{2}^{*}, \cdots, x_{n}^{*}\right)^{\top} \in \mathbb{X}^{*}$ such that

$$
x_{i}^{*}(t)=\left(\Phi x^{*}\right)_{i}(t)=c_{i}(t) x_{i}^{*}\left(t-\sigma_{i}(t)\right)+Y_{i}^{x_{i}^{*}}(t),
$$

and

$$
\begin{aligned}
x_{i}^{*}(t)= & c_{i}(t) x_{i}^{*}\left(t-\sigma_{i}(t)\right)+Y_{i}^{x_{i}^{*}}(t) \\
= & c_{i}(t) x_{i}^{*}\left(t-\sigma_{i}(t)\right)+\int_{-\infty}^{t} e_{-a_{i}}(t, \sigma(s))\left(-a_{i}(s) c_{i}(s) x_{i}^{*}\left(s-\sigma_{i}(s)\right)\right. \\
& +\sum_{j=1}^{n} b_{i j}(s) f_{j}\left(x_{j}^{*}\left(s-\tau_{i j}(s)\right)\right)+\sum_{j=1}^{n} d_{i j}(s) \mu_{j}(s) \\
& +\bigwedge_{j=1}^{n} \alpha_{i j}(s) \int_{s-\eta_{i j}(s)}^{s} g_{j}\left(x_{j}^{*}(u)\right) \Delta u+\bigvee_{j=1}^{n} \beta_{i j}(s) \int_{s-\xi_{i j}(s)}^{s} g_{j}\left(x_{j}^{*}(u)\right) \Delta u \\
& \left.+\bigwedge_{j=1}^{n} T_{i j}(s) \mu_{j}(s)+\bigvee_{j=1}^{n} s_{i j}(s) \mu_{j}(s)+I_{i}(s)\right) \Delta s, \quad i=1,2, \cdots, n .
\end{aligned}
$$

Hence

$$
\begin{aligned}
{\left[x_{i}^{*}(t)-c_{i}(t) x_{i}^{*}\left(t-\sigma_{i}(t)\right)\right]^{\Delta}=} & \left(Y_{i}^{x_{i}^{*}}(t)\right)^{\Delta} \\
= & -a_{i}(t) Y_{i}^{x_{i}^{*}}(t)-a_{i}(t) c_{i}(t) x_{i}^{*}\left(t-\sigma_{i}(t)\right) \\
& +\sum_{j=1}^{n} b_{i j}(t) f_{j}\left(x_{j}^{*}\left(t-\tau_{i j}(t)\right)\right)+\sum_{j=1}^{n} d_{i j}(t) \mu_{j}(t)
\end{aligned}
$$




$$
\begin{aligned}
& +\bigwedge_{j=1}^{n} \alpha_{i j}(t) \int_{t-\eta_{i j}(t)}^{t} g_{j}\left(x_{j}^{*}(s)\right) \Delta s+\bigvee_{j=1}^{n} \beta_{i j}(t) \int_{t-\xi_{i j}(t)}^{t} g_{j}\left(x_{j}^{*}(s)\right) \Delta s \\
& +\bigwedge_{j=1}^{n} T_{i j}(t) \mu_{j}(t)+\bigvee_{j=1}^{n} s_{i j}(t) \mu_{j}(t)+I_{i}(t) \\
& =-a_{i}(t) x_{i}^{*}(t)+\sum_{j=1}^{n} b_{i j}(t) f_{j}\left(x_{j}^{*}\left(t-\tau_{i j}(t)\right)\right)+\sum_{j=1}^{n} d_{i j}(t) \mu_{j}(t) \\
& +\bigwedge_{j=1}^{n} \alpha_{i j}(t) \int_{t-\eta_{i j}(t)}^{t} g_{j}\left(x_{j}^{*}(s)\right) \Delta s+\bigvee_{j=1}^{n} \beta_{i j}(t) \int_{t-\xi_{i j}(t)}^{t} g_{j}\left(x_{j}^{*}(s)\right) \Delta s \\
& +\bigwedge_{j=1}^{n} T_{i j}(t) \mu_{j}(t)+\bigvee_{j=1}^{n} s_{i j}(t) \mu_{j}(t)+I_{i}(t), \quad i=1,2, \cdots, n,
\end{aligned}
$$

so $x_{\mathfrak{i}}^{*}(t)$ is an anti-periodic solution of system (1.1). This completes the proof.

Theorem 3.2. Assume that $\left(\mathrm{H}_{1}\right)-\left(\mathrm{H}_{3}\right)$ hold, then system (1.1) has a unique anti-periodic solution that is globally exponentially stable.

Proof. From Theorem 3.1, we see that system (1.1) has an anti-periodic solution

$$
x^{*}(t)=\left(x_{1}^{*}(t), x_{2}^{*}(t), \cdots, x_{n}^{*}(t)\right)^{\top},
$$

with initial value $\psi^{*}(s)=\left(\psi_{1}^{*}(s), \psi_{2}^{*}(s), \cdots, \psi_{n}^{*}(s)\right)^{\top}$. Suppose that $x(t)=\left(x_{1}(t), x_{2}(t), \cdots, x_{n}(t)\right)^{\top}$ is an arbitrary solution of system (1.1) with initial value $\psi(s)=\left(\psi_{1}(s), \psi_{2}(s), \cdots, \psi_{n}(s)\right)^{\top}$ and

$$
z_{\mathfrak{i}}(\mathrm{t})=x_{\mathfrak{i}}(\mathrm{t})-x_{\mathfrak{i}}^{*}(t), \quad Z_{\mathfrak{i}}(t)=z_{\mathfrak{i}}(t)-c_{\mathfrak{i}}(t) z_{\mathfrak{i}}\left(t-\sigma_{\mathfrak{i}}(t)\right), \quad i=1,2, \cdots, n .
$$

Then

$$
\begin{aligned}
Z_{i}^{\Delta}(t)= & {\left[z_{i}(t)-c_{i}(t) z_{i}\left(t-\sigma_{i}(t)\right)\right]^{\Delta} } \\
= & -a_{i}(t) Z_{i}(t)-a_{i}(t) c_{i}(t) z_{i}\left(t-\sigma_{i}(t)\right)+\sum_{j=1}^{n} b_{i j}(t)\left(f_{j}\left(x_{j}\left(t-\tau_{i j}(t)\right)\right)\right. \\
& \left.-f_{j}\left(x_{j}^{*}\left(t-\tau_{i j}(t)\right)\right)\right)+\bigwedge_{j=1}^{n} \alpha_{i j}(t) \int_{t-\eta_{i j}(t)}^{t}\left(g_{j}\left(x_{j}(s)\right)-g_{j}\left(x_{j}^{*}(s)\right)\right) \Delta s \\
& +\bigvee_{j=1}^{n} \beta_{i j}(t) \int_{t-\xi_{i j}(t)}^{t}\left(g_{j}\left(x_{j}(s)\right)-g_{j}\left(x_{j}^{*}(s)\right)\right) \Delta s, \quad i=1,2, \cdots, n .
\end{aligned}
$$

From $\left(\mathrm{H}_{3}\right)$, there exists a constant $\lambda \in\left(0, \min _{1 \leqslant i \leqslant n}\left\{a_{i}^{-}\right\}\right)$such that $1-c_{i}^{+} \exp \left(\lambda \sigma_{i}^{+}\right)>0$, and

$$
\begin{aligned}
\max _{1 \leqslant i \leqslant n} & \left\{\frac { 1 } { ( 1 - c _ { i } ^ { + } \operatorname { e x p } ( \lambda \sigma _ { i } ^ { + } ) ) ( a _ { i } ^ { - } - \lambda ) } \left(\exp \left(\lambda \sigma_{i}^{+}\right) a_{i}^{+} c_{i}^{+}+\sum_{j=1}^{n} b_{i j}^{+} L_{j}^{f} \exp \left(\lambda \tau_{i j}^{+}\right)\right.\right. \\
& \left.\left.+\sum_{j=1}^{n} \alpha_{i j}^{+} L_{j}^{g} \eta_{i j}^{+} \exp \left(\lambda \eta_{i j}^{+}\right)+\sum_{j=1}^{n} \beta_{i j}^{+} L_{j}^{g} \xi_{i j}^{+} \exp \left(\lambda \xi_{i j}^{+}\right)\right)\right\}<1 .
\end{aligned}
$$

Denote $\|\varphi\|_{0}=\sup _{t \in[-\theta, 0]_{\mathbb{T}}} \max _{1 \leqslant i \leqslant n}\left|\left(\varphi_{i}(t)-x_{i}^{*}(t)\right)-c_{i}(t)\left(\varphi_{i}\left(t-\sigma_{i}(t)\right)-x_{i}^{*}\left(t-\sigma_{i}(t)\right)\right)\right|$ and

$$
\|Z(t)\|=\max _{1 \leqslant i \leqslant n}\left\{\left|Z_{i}(t)\right|\right\}
$$


Let $\varepsilon>0$ and $M>1$. Clearly,

$$
\|Z(0)\|<\left(\|\varphi\|_{0}+\varepsilon\right)
$$

and

$$
\|Z(t)\|<\left(\|\varphi\|_{0}+\varepsilon\right) e_{\ominus \lambda}\left(t, t_{0}\right)<M\left(\|\varphi\|_{0}+\varepsilon\right) e_{\ominus \lambda}\left(t, t_{0}\right), \quad \forall t \in[-\theta, 0]_{\mathbb{T}} .
$$

We claim that

$$
\|Z(t)\|<M\left(\|\varphi\|_{0}+\varepsilon\right) e_{\ominus \lambda}\left(t, t_{0}\right), \quad \forall t \in(0,+\infty)_{\mathbb{T}} .
$$

Contrarily, there exist a $t_{1} \in\left(t_{0},+\infty\right)_{\mathbb{T}}$ and some $i \in\{1,2, \cdots, n\}$ such that

$$
\left\{\begin{array}{l}
\left|Z_{i}\left(t_{1}\right)\right|=\left\|Z\left(t_{1}\right)\right\| \geqslant M\left(\|\varphi\|_{0}+\varepsilon\right) e_{\ominus \lambda}\left(t_{1}, t_{0}\right) \\
\|Z(t)\| \leqslant M\left(\|\varphi\|_{0}+\varepsilon\right) e_{\ominus \lambda}\left(t, t_{0}\right), t \in\left(t_{0}, t_{1}\right]_{\mathbb{T}} .
\end{array}\right.
$$

Therefore, there must exist a constant $c \geqslant 1$ such that

$$
\left\{\begin{array}{l}
\left|Z_{i}\left(t_{1}\right)\right|=\left\|Z\left(t_{1}\right)\right\|=c M\left(\|\varphi\|_{0}+\varepsilon\right) e_{\ominus \lambda}\left(t_{1}, t_{0}\right), \\
\|Z(t)\| \leqslant c M\left(\|\varphi\|_{0}+\varepsilon\right) e_{\ominus \lambda}\left(t, t_{0}\right), \quad t \in\left(t_{0}, t_{1}\right]_{\mathbb{T}} .
\end{array}\right.
$$

Moreover,

$$
\begin{aligned}
e_{\lambda}\left(\zeta, t_{0}\right)\left|z_{\mathfrak{i}}(\zeta)\right| & \leqslant e_{\lambda}\left(\zeta, t_{0}\right)\left|z_{\mathfrak{i}}(\zeta)-c_{\mathfrak{i}}(\zeta) z_{\mathfrak{i}}\left(\zeta-\sigma_{\mathfrak{i}}(\zeta)\right)\right|+e_{\lambda}\left(\zeta, \mathrm{t}_{0}\right)\left|c_{\mathfrak{i}}(\zeta) z_{\mathfrak{i}}\left(\zeta-\sigma_{\mathfrak{i}}(\zeta)\right)\right| \\
& \leqslant e_{\lambda}\left(\zeta, \mathrm{t}_{0}\right)\left|Z_{\mathfrak{i}}(\zeta)\right|+c_{i}^{+} \exp \left(\lambda \sigma_{i}^{+}\right) e_{\lambda}\left(\zeta-\sigma_{\mathfrak{i}}(\zeta), \mathrm{t}_{0}\right)\left|z_{\mathfrak{i}}\left(\zeta-\sigma_{\mathfrak{i}}(\zeta)\right)\right| \\
& \leqslant \operatorname{cM}\left(\|\varphi\|_{0}+\varepsilon\right)+c_{i}^{+} \exp \left(\lambda \sigma_{i}^{+}\right) \sup _{s \leqslant t} e_{\lambda}\left(s, t_{0}\right)\left|z_{\mathfrak{i}}(s)\right|
\end{aligned}
$$

for all $\zeta \leqslant t, t<t_{1}$, which implies that

$$
e_{\lambda}\left(t, t_{0}\right)\left|z_{i}(t)\right| \leqslant \sup _{s \leqslant t} e_{\lambda}\left(s, t_{0}\right)\left|z_{i}(s)\right| \leqslant \frac{c M\left(\|\varphi\|_{0}+\varepsilon\right)}{1-c_{i}^{+} \exp \left(\lambda \sigma_{i}^{+}\right)} .
$$

Integrating

$$
\begin{aligned}
& e_{-a_{i}}\left(t_{0}, \sigma(s)\right)\left(Z_{i}^{\Delta}(s)+a_{i}(s) Z_{i}(s)\right) \\
&=e_{-a_{i}}\left(t_{0}, \sigma(s)\right)\left\{-a_{i}(s) c_{i}(s) z_{i}\left(s-\sigma_{i}(s)\right)+\sum_{j=1}^{n} b_{i j}(s)\left(f_{j}\left(x_{j}\left(s-\tau_{i j}(s)\right)\right)\right.\right. \\
&\left.-f_{j}\left(x_{j}^{*}\left(s-\tau_{i j}(s)\right)\right)\right)+\bigwedge_{j=1}^{n} \alpha_{i j}(s) \int_{s-\eta_{i j}(s)}^{s}\left(g_{j}\left(x_{j}(s)\right)-g_{j}\left(x_{j}^{*}(s)\right)\right) \Delta s \\
&\left.+\bigvee_{j=1}^{n} \beta_{i j}(s) \int_{s-\xi_{i j}(s)}^{s}\left(g_{j}\left(x_{j}(s)\right)-g_{j}\left(x_{j}^{*}(s)\right)\right) \Delta s\right\}, \quad s \in\left[t_{0}, t\right]_{\mathbb{T}},
\end{aligned}
$$

we obtain

$$
\begin{aligned}
Z_{i}(t)= & Z_{i}\left(t_{0}\right) e_{-a_{i}}\left(t, t_{0}\right)+\int_{t_{0}}^{t} e_{-a_{i}}(t, \sigma(s))\left(-a_{i}(s) c_{i}(s) z_{i}\left(s-\sigma_{i}(s)\right)+\sum_{j=1}^{n} b_{i j}(s)\right. \\
& \times\left(f_{j}\left(x_{j}\left(s-\tau_{i j}(s)\right)\right)-f_{j}\left(x_{j}^{*}\left(s-\tau_{i j}(s)\right)\right)\right)+\bigwedge_{j=1}^{n} \alpha_{i j}(s) \int_{s-\eta_{i j}(s)}^{s}\left(g_{j}\left(x_{j}(u)\right)\right. \\
& \left.\left.-g_{j}\left(x_{j}^{*}(u)\right)\right) \Delta u+\bigvee_{j=1}^{n} \beta_{i j}(s) \int_{s-\xi_{i j}(s)}^{s}\left(g_{j}\left(x_{j}(u)\right)-g_{j}\left(x_{j}^{*}(u)\right)\right) \Delta u\right) \Delta s, t \in\left[t_{0}, t_{1}\right]_{\mathbb{T}} .
\end{aligned}
$$


Thus, $M>1$, (3.1), (3.2), (3.5) and (3.6) imply that

$$
\begin{aligned}
& \left|Z_{\mathfrak{i}}\left(t_{1}\right)\right|=\mid Z_{i}\left(t_{0}\right) e_{-a_{i}}\left(t_{1}, t_{0}\right)+\int_{t_{0}}^{t_{1}} e_{-a_{i}}\left(t_{1}, \sigma(s)\right)\left(-a_{i}(s) c_{i}(s) z_{i}\left(s-\sigma_{i}(s)\right)+\sum_{j=1}^{n} b_{i j}(s)\right. \\
& \times\left(f_{j}\left(x_{j}\left(s-\tau_{i j}(s)\right)\right)-f_{j}\left(x_{j}^{*}\left(s-\tau_{i j}(s)\right)\right)\right)+\bigwedge_{j=1}^{n} \alpha_{i j}(s) \int_{s-\eta_{i j}(s)}^{s}\left(g_{j}\left(x_{j}(u)\right)\right. \\
& \left.\left.-g_{j}\left(x_{j}^{*}(u)\right)\right) \Delta u+\bigvee_{j=1}^{n} \beta_{i j}(s) \int_{s-\xi_{i j}(s)}^{s}\left(g_{j}\left(x_{j}(u)\right)-g_{j}\left(x_{j}^{*}(u)\right)\right) \Delta u\right) \Delta s \mid \\
& \leqslant\left|Z_{i}\left(t_{0}\right)\right| e_{-a_{i}}\left(t_{1}, t_{0}\right)+\int_{t_{0}}^{t_{1}} e_{-a_{i}}\left(t_{1}, \sigma(s)\right) \mid-a_{i}(s) c_{i}(s) z_{i}\left(s-\sigma_{i}(s)\right)+\sum_{j=1}^{n} b_{i j}(s) \\
& \times\left(f_{j}\left(x_{j}\left(s-\tau_{i j}(s)\right)\right)-f_{j}\left(x_{j}^{*}\left(s-\tau_{i j}(s)\right)\right)\right)+\bigwedge_{j=1}^{n} \alpha_{i j}(s) \int_{s-\eta_{i j}(s)}^{s}\left(g_{j}\left(x_{j}(u)\right)\right. \\
& \left.-g_{j}\left(x_{j}^{*}(u)\right)\right) \Delta u+\bigvee_{j=1}^{n} \beta_{i j}(s) \int_{s-\xi_{i j}(s)}^{s}\left(g_{j}\left(x_{j}(u)\right)-g_{j}\left(x_{j}^{*}(u)\right)\right) \Delta u \mid \Delta s \\
& \leqslant\left|Z_{i}\left(t_{0}\right)\right| e_{-a_{i}}\left(t_{1}, t_{0}\right)+\int_{t_{0}}^{t_{1}} e_{-a_{i}}\left(t_{1}, \sigma(s)\right)\left(a_{i}^{+} c_{i}^{+}\left|z_{i}\left(s-\sigma_{i}(s)\right)\right|+\sum_{j=1}^{n} b_{i j}^{+} L_{j}^{f}\right. \\
& \times\left|x_{j}\left(s-\tau_{i j}(s)\right)-x_{j}^{*}\left(s-\tau_{i j}(s)\right)\right|+\sum_{j=1}^{n} \alpha_{i j}^{+} L_{j}^{g} \int_{s-\eta_{i j}(s)}^{s}\left|x_{j}(u)-x_{j}^{*}(u)\right| \Delta u \\
& \left.+\sum_{j=1}^{n} \beta_{i j}^{+} L_{j}^{g} \int_{s-\xi_{i j}(s)}^{s}\left|x_{j}(u)-x_{j}^{*}(u)\right| \Delta u\right) \Delta s \\
& \leqslant\left(\|\varphi\|_{0}+\varepsilon\right) e_{\ominus \lambda}\left(t_{1}, t_{0}\right) e_{-a_{i} \oplus \lambda}\left(t_{1}, t_{0}\right)+\int_{t_{0}}^{t_{1}} e_{-a_{i} \oplus \lambda}\left(t_{1}, \sigma(s)\right)\left(\frac{\exp \left(\lambda \sigma_{i}^{+}\right)}{1-c_{i}^{+} \exp \left(\lambda \sigma_{i}^{+}\right)} a_{i}^{+} c_{i}^{+}\right. \\
& +\sum_{j=1}^{n} b_{i j}^{+} L_{j}^{f} \frac{\exp \left(\lambda \tau_{i j}^{+}\right)}{1-c_{i}^{+} \exp \left(\lambda \sigma_{i}^{+}\right)}+\sum_{j=1}^{n} \alpha_{i j}^{+} L_{j}^{g} \eta_{i j}^{+} \frac{\exp \left(\lambda \eta_{i j}^{+}\right)}{1-c_{i}^{+} \exp \left(\lambda \sigma_{i}^{+}\right)} \\
& \left.+\sum_{j=1}^{n} \beta_{i j}^{+} L_{j}^{g} \xi_{i j}^{+} \frac{\exp \left(\lambda \xi_{i j}^{+}\right)}{1-c_{i}^{+} \exp \left(\lambda \sigma_{i}^{+}\right)}\right) \Delta \operatorname{scM}\left(\|\varphi\|_{0}+\varepsilon\right) e_{\ominus \lambda}\left(t_{1}, t_{0}\right) \\
& \leqslant\left(\|\varphi\|_{0}+\varepsilon\right) e_{\ominus \lambda}\left(t_{1}, t_{0}\right) e_{-a_{i} \oplus \lambda}\left(t_{1}, t_{0}\right)+\frac{1-e_{-a_{i}^{-} \oplus \lambda}\left(t_{1}, t_{0}\right)}{a_{i}^{-}-\lambda}\left(\frac{\exp \left(\lambda \sigma_{i}^{+}\right)}{1-c_{i}^{+} \exp \left(\lambda \sigma_{i}^{+}\right)} a_{i}^{+} c_{i}^{+}\right. \\
& +\sum_{j=1}^{n} b_{i j}^{+} L_{j}^{f} \frac{\exp \left(\lambda \tau_{i j}^{+}\right)}{1-c_{i}^{+} \exp \left(\lambda \sigma_{i}^{+}\right)}+\sum_{j=1}^{n} \alpha_{i j}^{+} L_{j}^{g} \eta_{i j}^{+} \frac{\exp \left(\lambda \eta_{i j}^{+}\right)}{1-c_{i}^{+} \exp \left(\lambda \sigma_{i}^{+}\right)} \\
& \left.+\sum_{j=1}^{n} \beta_{i j}^{+} L_{j}^{g} \xi_{i j}^{+} \frac{\exp \left(\lambda \xi_{i j}^{+}\right)}{1-c_{i}^{+} \exp \left(\lambda \sigma_{i}^{+}\right)}\right) \operatorname{cM}\left(\|\varphi\|_{0}+\varepsilon\right) e_{\ominus \lambda}\left(t_{1}, t_{0}\right) \\
& \leqslant c M\left(\|\varphi\|_{0}+\varepsilon\right) e_{\ominus \lambda}\left(t_{1}, t_{0}\right)\left(\left(\frac{1}{c M}-1\right) e_{-a_{i}^{-} \oplus \lambda}\left(t_{1}, t_{0}\right)+1\right) \\
& <\operatorname{cM}\left(\|\varphi\|_{0}+\varepsilon\right) e_{\ominus \lambda}\left(t_{1}, t_{0}\right) \text {, }
\end{aligned}
$$

which contradicts the first equation of (3.4). Therefore, (3.3) holds. Letting $\varepsilon \rightarrow 0^{+}$, we get $\|Z(t)\| \leqslant M\|\varphi\|_{0} e_{\ominus \lambda}\left(t, t_{0}\right), \quad \forall t \in\left(t_{0},+\infty\right)_{\mathbb{T}}$. 
Hence, the anti-periodic solution of system (1.1) is globally exponentially stable and the uniqueness follows from the stability. The proof is complete.

Remark 3.3. From the conditions of Theorems 3.1 and 3.2, it is easy to see that both the continuous time case and the discrete time case of FCNNs (1.1) have the same anti-periodic dynamics.

\section{An example}

In this section, we give an example to illustrate the feasibility and effectiveness of our results obtained in Section 3.

Example 4.1. In (1.1), let $\mathrm{n}=2$, and the coefficients are taken as follows:

$$
\begin{aligned}
f_{1}(x)=\frac{1}{18} \sin ^{3} x, \quad f_{2}(x)=\frac{1}{6} \sin x, \quad g_{1}(x)=\frac{1}{12} \sin 2 x, \quad g_{2}(x)=\frac{1}{18} \sin 3 x, \\
I_{1}(t)=5 \sin \frac{t}{2}, \quad I_{2}(t)=\sin ^{3} \frac{t}{2^{\prime}} \quad c_{1}(t)=0.1 \sin t, \quad c_{2}(t)=\frac{1}{15} \cos t, \\
\left.\left.a_{1}(t)=0.8+0.1 \mid \sin t\right), \quad a_{2}(t)=0.7+0.1 \mid \cos t\right), \quad b_{11}(t)=0.4+0.1 \sin t, \\
b_{12}(t)=0.3+0.1 \cos t, \quad b_{21}(t)=0.5+0.1 \cos t, \quad b_{22}(t)=0.2+0.1 \sin t, \\
d_{11}(t)=0.2-0.1 \cos t, \quad d_{12}(t)=0.3+0.1 \sin t, \quad d_{21}(t)=0.6-0.1 \cos t, \\
d_{22}(t)=0.5-0.1 \sin t, \quad \alpha_{11}(t)=0.5+0.1 \cos t, \quad \alpha_{12}(t)=0.3+0.2 \sin t, \\
\alpha_{21}(t)=0.4+0.1 \sin t, \quad \alpha_{22}(t)=0.3-0.1 \sin t, \quad \beta_{11}(t)=0.1+0.3 \sin t, \\
\beta_{12}(t)=0.5+0.1 \cos t, \quad \beta_{21}(t)=0.3+0.2 \cos t, \quad \beta_{22}(t)=0.1+0.4 \sin t, \\
\sigma_{1}(t)=|\cos t|, \quad \sigma_{2}(t)=|\sin t|, \quad \tau_{11}(t)=2|\cos t|, \quad \tau_{12}(t)=|\cos t|, \quad \tau_{21}(t)=3|\sin t|, \\
\left.\tau_{22}(t)=5|\cos t|, \quad \eta_{11}(t)=2|\cos t|, \quad \eta_{12}(t)=|\sin t|, \quad \eta_{21}(t)=\mid \sin t\right), \quad \eta_{22}(t)=2|\sin t|, \\
\mu_{1}(t)=\sin \frac{t}{2}, \quad \mu_{2}(t)=-\sin ^{3} \frac{t}{2}, \quad \xi_{i j}(t)=|\sin t|, \quad T_{i j}(t)=5 \cos ^{2} t, \quad s_{i j}(t)=3 \sin { }^{3} t, \quad i, j=1,2 .
\end{aligned}
$$

Obviously, conditions $\left(\mathrm{H}_{1}\right)$ and $\left(\mathrm{H}_{2}\right)$ are verified. By simple calculation, we have

$$
\begin{gathered}
\mathrm{L}_{1}^{\mathrm{f}}=\mathrm{L}_{2}^{\mathrm{f}}=\mathrm{L}_{1}^{\mathrm{g}}=\mathrm{L}_{2}^{\mathrm{g}}=\frac{1}{6}, \quad \mathrm{c}_{1}^{+}=\frac{1}{10}, \quad \mathrm{c}_{2}^{+}=\frac{1}{15}, \quad \mathrm{a}_{1}^{+}=0.9, \quad \mathrm{a}_{2}^{+}=0.8, \quad a_{1}^{-}=0.8, \quad \mathrm{a}_{2}^{-}=0.7, \\
\mathrm{~b}_{11}^{+}=0.5, \quad \mathrm{~b}_{12}^{+}=0.4, \quad \mathrm{~b}_{21}^{+}=0.6, \quad \mathrm{~b}_{22}^{+}=0.3, \quad \alpha_{11}^{+}=0.6, \quad \alpha_{12}^{+}=0.5, \quad \alpha_{21}^{+}=0.5, \\
\alpha_{22}^{+}=0.4, \quad \beta_{11}^{+}=0.4, \quad \beta_{12}^{+}=0.6, \quad \beta_{21}^{+}=0.5, \quad \beta_{22}^{+}=0.5, \quad \sigma_{1}^{+}=1, \quad \sigma_{2}^{+}=1, \\
\tau_{11}^{+}=2, \quad \tau_{12}^{+}=1, \quad \tau_{21}^{+}=3, \quad \tau_{22}^{+}=5, \quad \eta_{11}^{+}=2, \quad \eta_{12}^{+}=\eta_{21}^{+}=1, \quad \eta_{22}^{+}=2, \quad \xi_{11}^{+}=\xi_{12}^{+}=\xi_{21}^{+}=\xi_{22}^{+}=1,
\end{gathered}
$$
and

$$
\begin{aligned}
\rho & =\max _{1 \leqslant i \leqslant 2}\left\{c_{i}^{+}+\frac{1}{a_{i}^{-}}\left(a_{i}^{+} c_{i}^{+}+\sum_{j=1}^{2}\left(L_{j}^{f} b_{i j}^{+}+L_{j}^{g} \alpha_{i j}^{+} \eta_{i j}^{+}+L_{j}^{g} \beta_{i j}^{+} \xi_{i j}^{+}\right)\right)\right\} \\
& =\max \left\{\frac{231}{240}, \frac{19}{21}\right\}<1
\end{aligned}
$$

so condition $\left(\mathrm{H}_{3}\right)$ is also satisfied. Therefore, according to Theorems 3.1 and 3.2, system (1.1) has a unique $2 \pi$-antiperiodic solution that is globally exponentially stable (see Figures 1-4). 

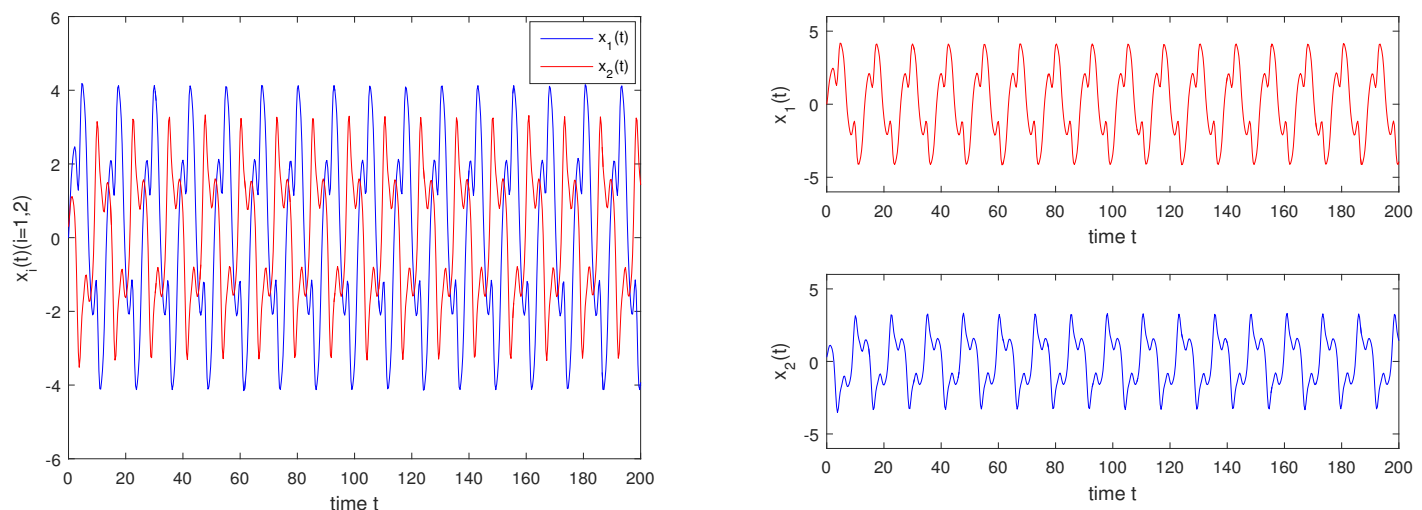

Figure 1: When $\mathbb{T}=\mathbb{R}$, anti-periodic solution of system (1.1) with initial condition $\left(x_{1}(0), x_{2}(0)\right)^{\top}=(0.25,0.32)^{\top}$.

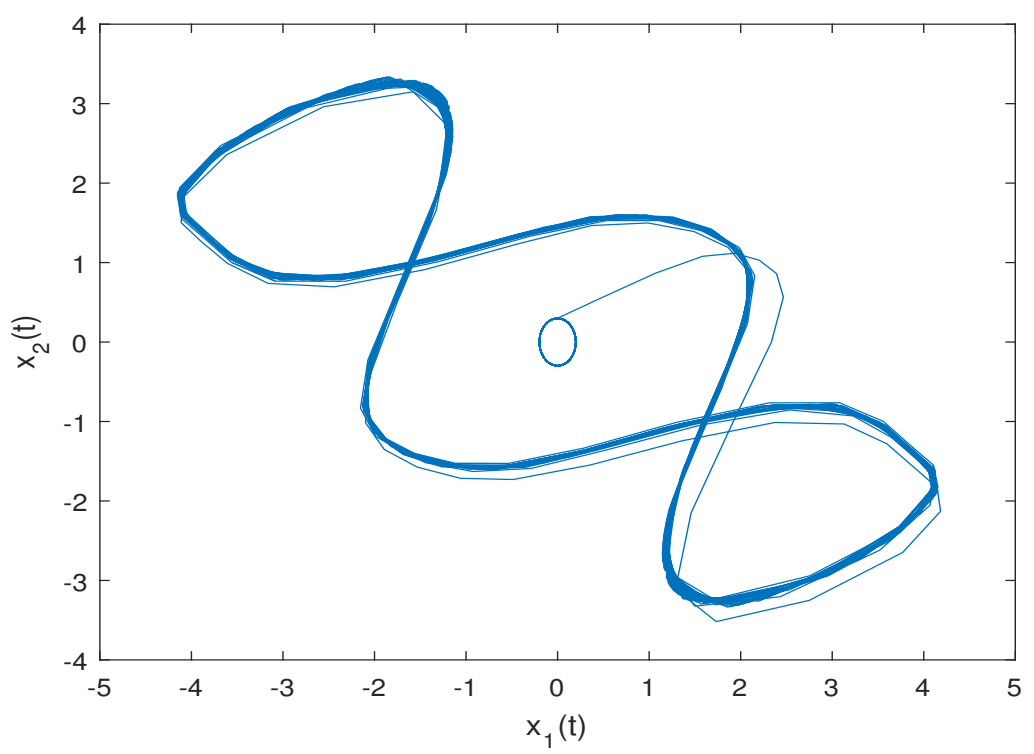

Figure 2: When $\mathbb{T}=\mathbb{R}$, phase response of state variables $x_{1}(t)$ and $x_{2}(t)$ of system (1.1) with initial condition $\left(x_{1}(0), x_{2}(0)\right)^{\mathrm{T}}=$ $(0.25,0.32)^{\mathrm{T}}$.
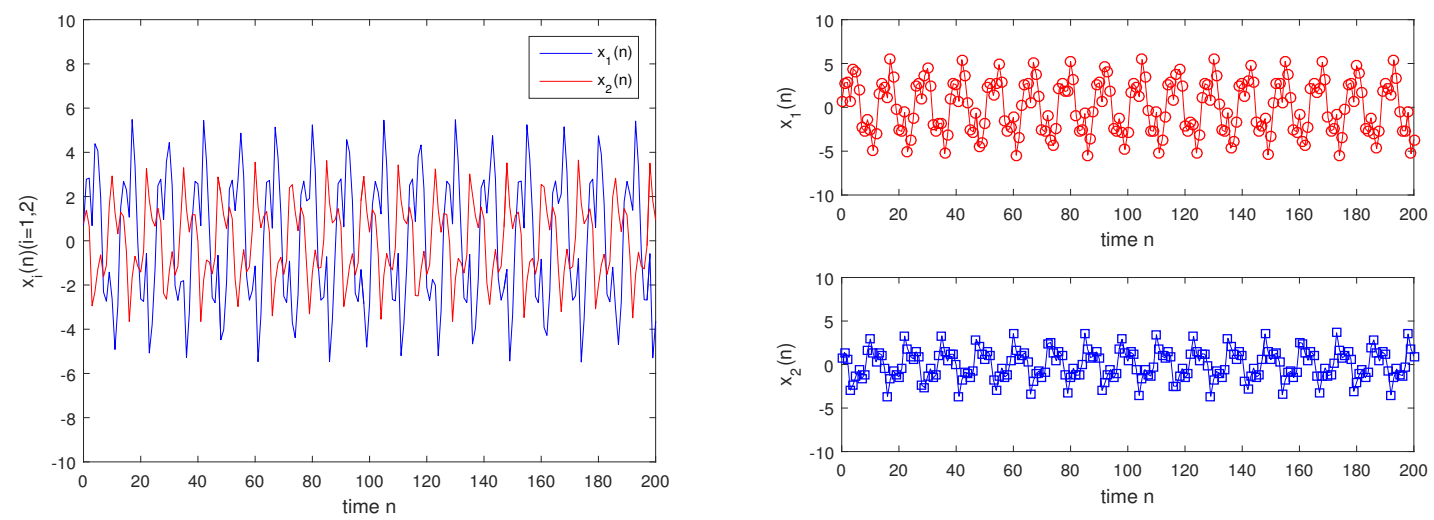

Figure 3: When $\mathbb{T}=\mathbb{Z}$, anti-periodic solution of system (1.1) with initial condition $(0.72,0.85)$. 


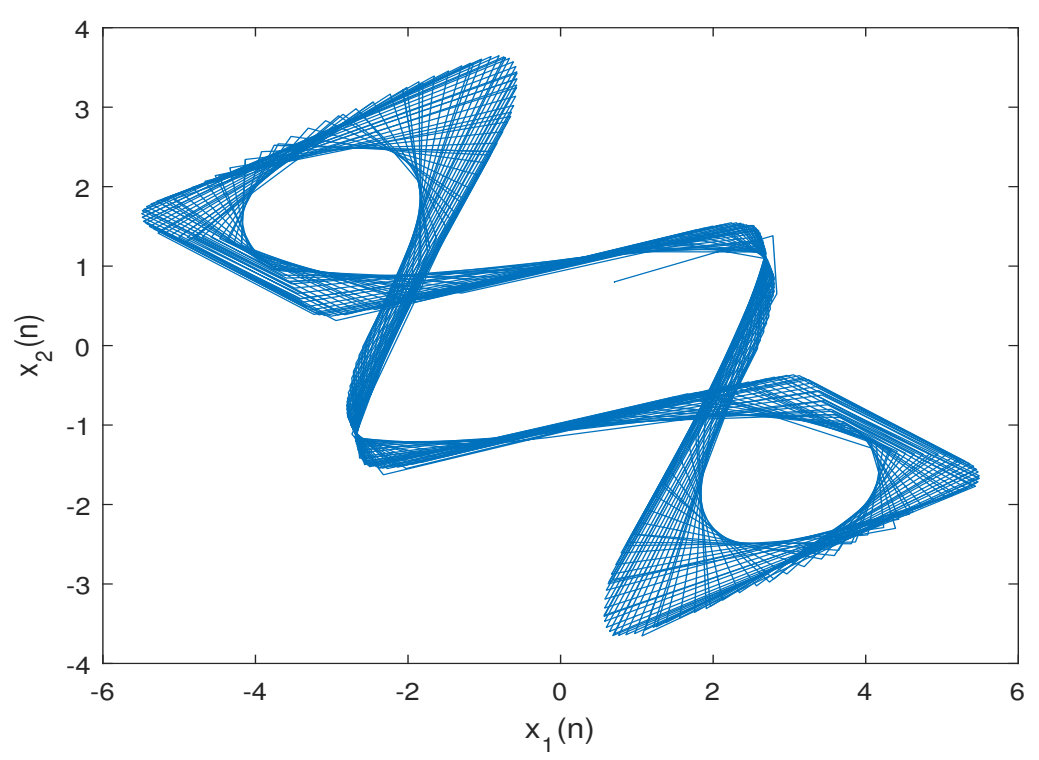

Figure 4: When $\mathbb{T}=\mathbb{Z}$, phase response of state variables $x_{1}(n)$ and $x_{2}(n)$ of system (1.1) with initial condition $(0.72,0.85)$.

\section{Conclusion}

In this paper, we have investigated the FCNNs with mixed time-varying delays and D operators on time scales. By using the Banach's fixed point theorem and the theory of calculus on time scales, we obtain some sufficient conditions for the existence and exponential stability of anti-periodic solutions for FCNNs. An example has been given to demonstrate the effectiveness of our results. The method of this paper can be applied to study other neural networks with D operators on times scales.

\section{Acknowledgment}

This work is supported by the National Natural Sciences Foundation of People's Republic of China under Grant 11361072.

\section{References}

[1] P. Balasubramaniam, V. Vembarasan, Robust stability of uncertain fuzzy BAM neural networks of neutral-type with Markovian jumping parameters and impulses, Comput. Math. Appl., 62 (2011), 1838-1861. 1

[2] H.-M. Bao, Existence and exponential stability of periodic solution for BAM fuzzy CohenGrossberg neural networks with mixed delays, Neural Proc. Lett., 43 (2016), 871-885. 1

[3] M. Bohner, A. Peterson, Dynamic equations on time scales, An introduction with applications. Birkhuser Birkhäuser Boston, Inc., Boston, MA, (2001). 1

[4] L. O. Chua, L. Yang, Cellular neural networks: applications, IEEE Trans. Circuits and Systems, 35 (1988), $1273-1290$. 1

[5] L. O. Chua, L. Yang, Celluar neural networks: theory, IEEE Trans. Circuits and Systems, 35 (1988), 1257-1272. 1

[6] Z.-D. Huang, Almost periodic solutions for fuzzy cellular neural networks with time-varying delays, Neural Comput. Appl., 28 (2017), 2313-2320. 1

[7] R.-W. Jia, Finite-time stability of a class of fuzzy cellular neural networks with multi-proportional delays, Fuzzy Sets and Systems, 319 (2017), 70-80. 1

[8] Y.-K. Li, X.-R. Chen, L. Zhao, Stability and existence of periodic solutions to delayed CohenGrossberg BAM neural networks with impulses on time scales, Neurocomputing, 72 (2009), 1621-1630. 1

[9] Y.-K. Li, C. Wang, Uniformly almost periodic functions and almost periodic solutions to dynamic equations on time scales, Abstr. Appl. Anal., 2011 (2011), 22 pages. 2.2

[10] Y.-K. Li, C. Wang, Existence and global exponential stability of equilibrium for discrete-time fuzzy BAM neural networks with variable delays and impulses, Fuzzy Sets and Systems, 217 (2013), 62-79. 1 
[11] Y.-K. Li, L. Yang, W.-Q. Wu, Periodic solutions for a class of fuzzy BAM neural networks with distributed delays and variable coefficients, Internat. J. Bifur. Chaos Appl. Sci. Engrg., 20 (2010), 1551-1565.

[12] Y.-K. Li, T.-W. Zhang, Global exponential stability of fuzzy interval delayed neural networks with impulses on time scales, Int. J. Neural Syst., 19 (2009), 449-456.

[13] B.-W. Liu, Global exponential stability for BAM neural networks with time-varying delays in the leakage terms, Nonlinear Anal. Real World Appl., 14 (2013), 559-566.

[14] Y. Luo, W.-B. Wang, J.-H. Shen, Existence of positive periodic solutions for two kinds of neutral functional differential equations, Appl. Math. Lett., 21 (2008), 581-587.

[15] C.-J. Xu, Q.-M. Zhang, Y.-S. Wu, Existence and exponential stability of periodic solution to fuzzy cellular neural networks with distributed delays, Int. J. Fuzzy Syst., 18 (2016), 41-51. 1

[16] T. Yang, L.-B. Yang, The global stability of fuzzy cellular neural network, IEEE Trans. Circuits Systems I Fund. Theory Appl., 43 (1996), 880-883. 1, 2.1

[17] T. Yang, L.-B. Yang, C.-W. Wu, L. O. Chua, Fuzzy celluar neural networks: applications, Fourth IEEE International Workshop on Cellular Neural Networks and their Applications Proceedings, Seville, Spain, (CNNA-96), (1996), 225-230. 1

[18] T. Yang, L.-B. Yang, C.-W. Wu, L. O. Chua, Fuzzy cellular neural networks: theory, Fourth IEEE International Workshop on Cellular Neural Networks and their Applications Proceedings, Seville, Spain, (CNNA-96), (1996), 181186. 1

[19] K. Yuan, J.-D. Cao, J.-M. Deng, Exponential stability and periodic solutions of fuzzy cellular neural networks with timevarying delays, Neurocomputing, 69 (2006), 1619-1627. 1

[20] Q.-H. Zhang, R.-G. Xiang, Global asymptotic stability of fuzzy cellular neural networks with time-varying delays, Phys. Lett. A, 372 (2008), 3971-3977. 1

[21] L.-L. Zhao, Y.-K. Li, Existence and exponential stability of anti-periodic solutions of high-order Hopfield neural networks with delays on time scales, Differ. Equ. Dyn. Syst., 19 (2011), 13-26. 2.4

[22] L.-Q. Zhou, Global asymptotic stability of cellular neural networks with proportional delays, Nonlinear Dynam., 77 (2014), 41-47. 1

[23] L.-Q. Zhou, X.-B. Chen, Y.-X. Yang, Asymptotic stability of cellular neural networks with multiple proportional delays, Appl. Math. Comput., 229 (2014), 457-466. 1 